\title{
Designed, Flexible Electrochromic Display Device with Fe(II)-Based Metallo-Supramolecular Polymer Using Mechanically Etched ITO Film
}

\author{
Masayoshi Higuchi* and Yukio Fujii \\ Electronic Functional Macromolecules Group, Research Center for Functional Materials, \\ National Institute for Materials Science, 1-1 Namiki, Tsukuba, Ibaraki 305-0044, Japan \\ *HIGUCHI.Masayoshi@nims.go.jp
}

\begin{abstract}
Electrochromic (EC) displays are expected as energy-saving smart windows or digital signages because of the memory property. We succeeded in developing a designed and flexible electrochromic (EC) display device with $\mathrm{Fe}(\mathrm{II})$-based metallo-supramolecular polymer (polyFe) using a mechanically etched ITO film. Mechanical etching an ITO film was performed with a cutting machine having a blade of which length was adjusted so as not to cut off the film. The fabricated EC device was composed of the etched ITO film, a polyFe layer, an electrolyte layer, a nickel hexacyanoferrate layer as the counter material, and another ITO film. The EC device showed unique changes of the display upon applying a voltage of $1.5 \mathrm{~V}$ between the two ITO electrodes. It was revealed that the EC changes were controlled by the etching pattern such as a closed circle or the formation of a narrow ITO path.

Keywords: Electrochromic display, Metallo-supramolecular polymer, Mechanical etching, Design, Flexible
\end{abstract}

\section{Introduction}

Electrochromic (EC) materials have attracted much attention because of the potential applications such as electrical dimming glass in office and antiglare mirror in cars [1-7]. Especially, flexible EC devices (ECDs) are expected to expand the application fields to displays or fashion [8-14]. Tungsten oxide and viologens are representative EC materials but, they are not always suitable to the flexible ECDs. Plastic film with an ITO layer (ITO film) is the required component in the flexible ECDs, but the ITO film is not proper to vapor deposition process at high temperature, which is necessary to prepare a layer of inorganic EC materials such as tungsten oxide, because of the low thermal stability. The ITO film is not also appropriate to fabricating ECDs with organic molecules containing viologens, because the flexibility of the ITO films tends to cause leaking a solution of the EC molecule, which is injected to the cell, from the sealed part of the fabricated ECD.

One of the best coating methods on the ITO film at low temperature is spin- or spray-coating a solution of EC material. From the viewpoint, metallo-supramolecular polymers (MSPs) are good candidates as the EC material in flexible ECDs, because the polymers have high solubility in polar solvent such as water and methanol. MSPs are composed of metal ion and multi-topic organic ligand. They are expected to show unique electronic properties because of the electronic interaction between the metal and the ligand $[15,16]$.

$\mathrm{Fe}(\mathrm{II})$-based MSP composed of $\mathrm{Fe}(\mathrm{II})$ ion and bis(terpyridyl)benzene (polyFe) shows EC properties triggered by the redox between $\mathrm{Fe}(\mathrm{II})$ and Fe(III) (Fig. 1) [17,18].

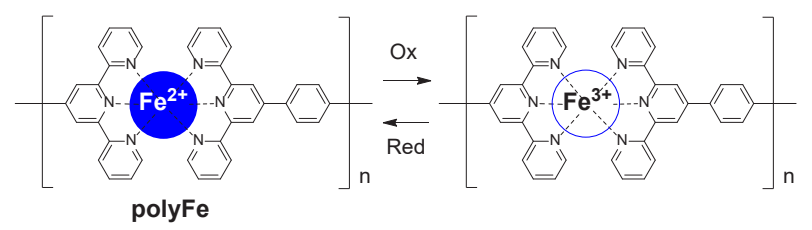

Fig. 1. Redox of polyFe.

PolyFe with $\mathrm{Fe}(\mathrm{II})$ (reduced state) has bluish purple color caused by the metal-to-ligand charge 
transfer (MLCT) absorption around $580 \mathrm{~nm}$. The color becomes colorless by electrochemical oxidation of $\mathrm{Fe}$ (II) to $\mathrm{Fe}(\mathrm{III})$ in polyFe (oxidized state), because of the disappearance of the MLCT absorption. The appearance and disappearance of the MLCT absorption by the redox between Fe(II) and $\mathrm{Fe}(\mathrm{III})$ can be explained by the energy diagram as shown in Fig. 2. It is considered that the $\mathrm{d}$ electron of $\mathrm{Fe}(\mathrm{II})$ contributed to the MLCT absorption is removed electrochemically in the oxidized state of polyFe then the MLCT absorption is vanished.

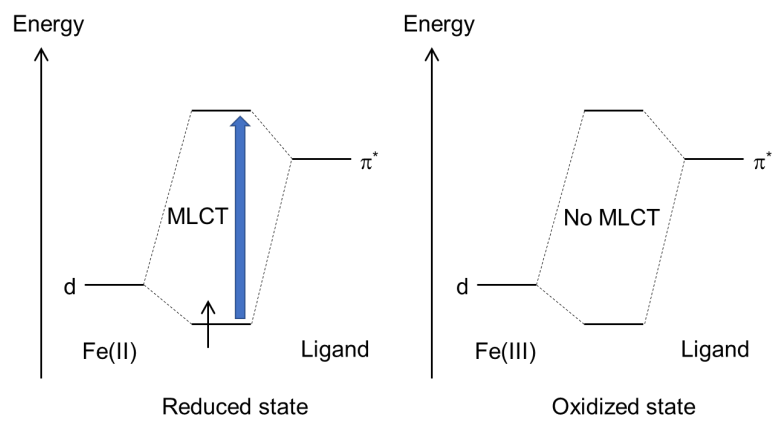

Fig. 2. Energy diagrams of the reduced and oxidized states in polyFe.

A general device structure with MSP is shown in Fig. 3 [19,20]. The electrolyte layer provides anion to the MSP layer when the MSP is oxidized electrochemically. The counter material layer is introduced to accept the complementary redox in the device, which is accompanied with the redox of MSP. Plastic is available as the substrate, because MSPs can be coated on an ITO layer by spin- or spray-coating.

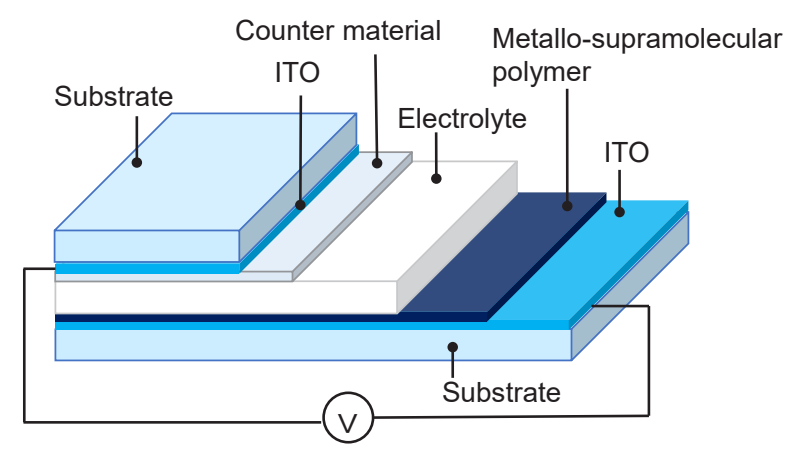

Fig. 3. A device structure of ECDs with MSP.

Here we report fabrication of a designed ECD with polyFe by mechanical etching an ITO film and the EC properties.

\section{Experimental}

\subsection{Chemicals}

All reagents and solvents were of high pure analytical grade and used without further purification. PolyFe was purchased from NARD institute, Ltd. Indium tin oxide (ITO)-coated plastic sheets were provided from Nitto Denko Co. Poly(methyl methacrylate) (PMMA) was obtained from Tokyo Chemical Industry Co. (M0088). Battery-grade propylene carbonate (PC), batterygrade $\mathrm{LiClO}_{4}$, and spectrochemical analysis-grade methanol $(\mathrm{MeOH})$ were supplied by Fujifilm Wako Chemicals Co. Nickel hexacyanoferrate (NiHCF) was got from Kanto Chemical Co.

\subsection{Equipment}

A cutting machine (Roland Desktop Cutter STIKA SV-15) was used to scratch the ITO film (Fig. 4). Spray coater (API-40RA, apeiros) was utilized to prepare thin layers of polyFe and NiHCF on ITO films.

\subsection{Gel electrolyte}

The gel nonaqueous electrolyte was prepared by the mixing of PMMA, PC, and $\mathrm{LiClO}_{4}$ $\left[\mathrm{PMMA} / \mathrm{PC} / \mathrm{LiClO}_{4}=20: 20: 3 \quad(\mathrm{w} / \mathrm{w})\right]$ with continuous stirring.

\subsection{EC device fabrication}

(i) Mechanical etching was performed on an ITO film (size: $10 \times 10 \mathrm{~cm}$; film thickness: $150 \mu \mathrm{m}$ ) using the cutting machine with the blade of which length was adjusted so as not to cut off the film. The depth of scratching was controlled to be about 50 $\mu \mathrm{m}$. The film was used as an electrode of a polyFe layer.

(ii) The mechanically etched ITO film and another ITO film, which was used as the counter electrode, were fixed on flat glass substrates for spray-coating (Fig. 4a).

(iii) A polyFe layer was prepared on the mechanically etched ITO film by spray-coating a methanol solution of polyFe $(8.3 \mathrm{~g} / \mathrm{mL})$ (Fig. $4 \mathrm{~b})$. A $\mathrm{NiHCF}$ layer as the counter material was formed on another ITO film by spray-coating an aqueous solution of NiHCF $(4.2 \mathrm{mg} / \mathrm{mL})$.

(iv) A prepared gel electrolyte was dropped on the $\mathrm{NiHCF}$ layer and the two ITO films with the polyFe or NiHCF layer were stacked face-to-face through the gel electrolyte. Then, the two supporting glass substrates outside were clipped to fix the stacked layers. The assembled device was heated at $98{ }^{\circ} \mathrm{C}$ for $5 \mathrm{~min}$. During the heating, the electrolyte 
became transparent and physically glued the two ITO films. Finally, the flexible ECD with polyFe was obtained by the removal of the supporting glass substrates.
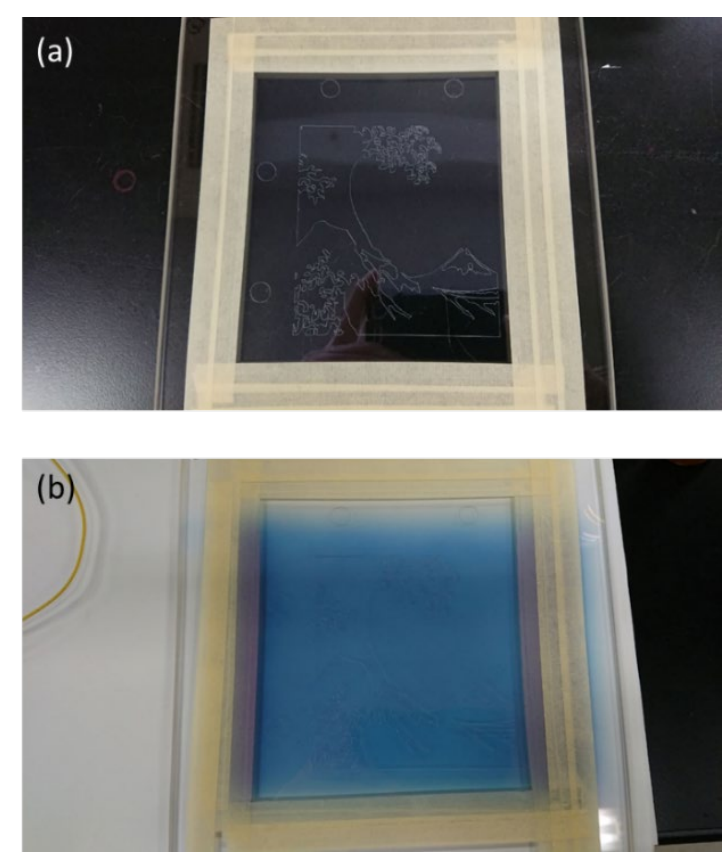

Fig. 4. (a) The mechanically etched ITO film, which was fixed on a glass substrate for spray coating. (b) The spraycoated polyFe layer on the ITO film.

\section{Results and discussion}

\subsection{Strategy}

Strategies to display an image on an ECD are shown in Fig. 5.

\section{(a) Drawing an image with MSP}

The simplest way to display an image on an ECD is to draw the image with MSP (Fig. 5a). The colored image becomes colorless by applying a voltage between the two ITO electrodes, because the MSP is oxidized electrochemically.

(b) Pasting a designed transparent insulating film

Inserting a transparent insulating film between the MSP layer and the ITO layer is another way to display an image on the ECD. When an oxidative potential is applied to the MSP layer, the part covered with the insulating film cannot change the color, because the anion transfer from the electrolyte layer to the MSP layer is prevented by the insulating film. As the result, the shape of the insulating film appears, because the rest uncovered part becomes colorless (Fig. 5b).

(c) Mechanical etching an ITO film (this work) Chemical or physical etching is a useful method to process electrode in electronics. In ECDs, mechanical etching (= scratching) the electrode is expected to provide a unique display, because the etched lines prevent electron transfer on the ITO layer (Fig. 5c).
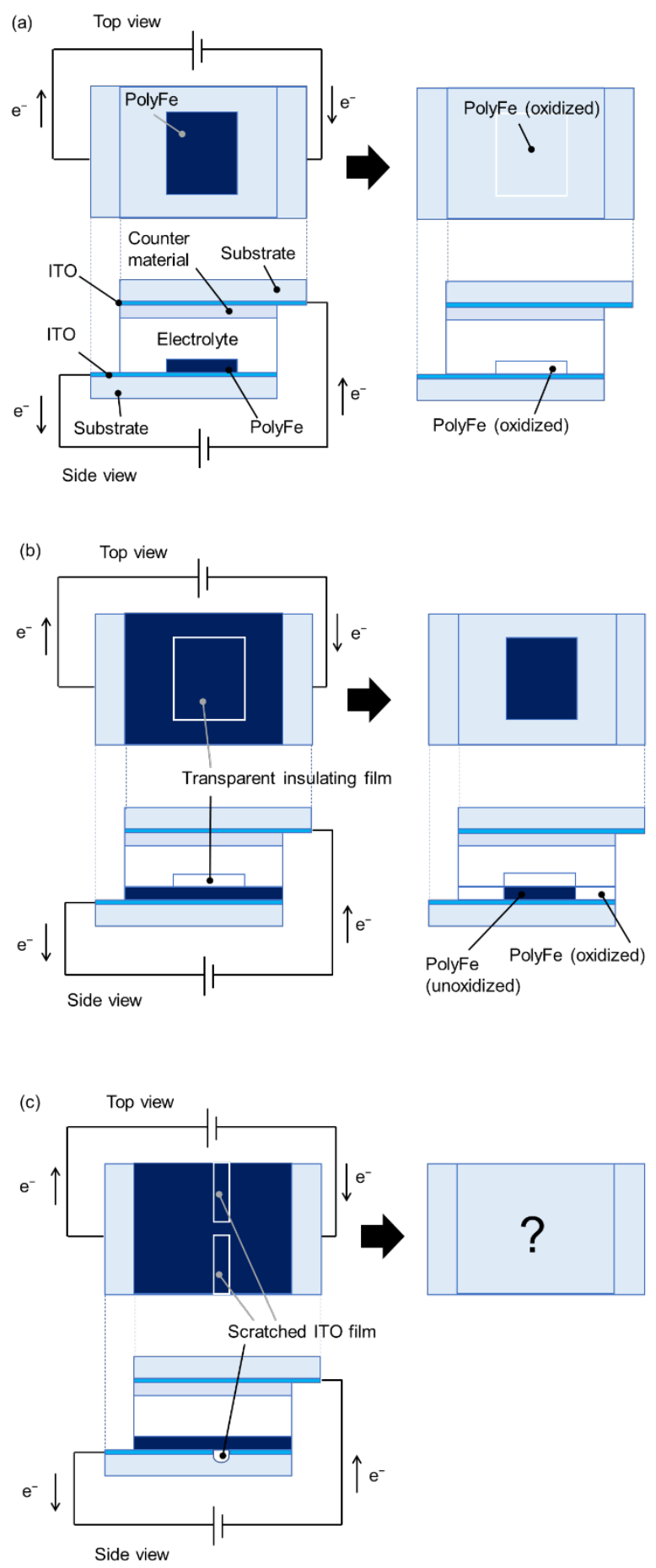

Fig. 5. Strategies to display an image on an ECD. (a) drawing an image with MSP, (b) displaying an image by inserting a transparent insulating film, and (c) this work (scratching an ITO film). 


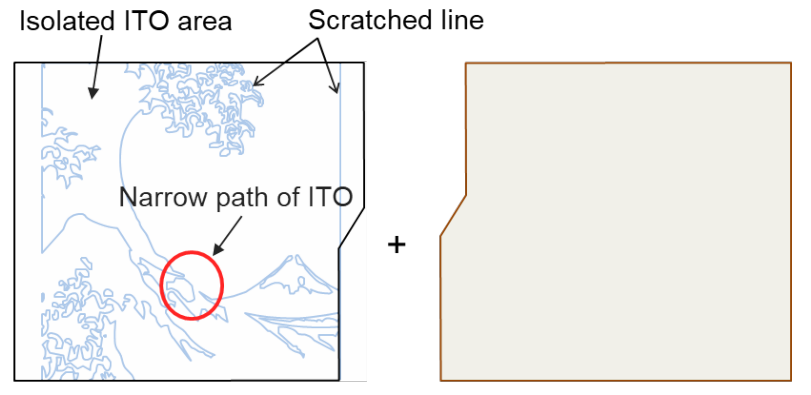

EC Electrode
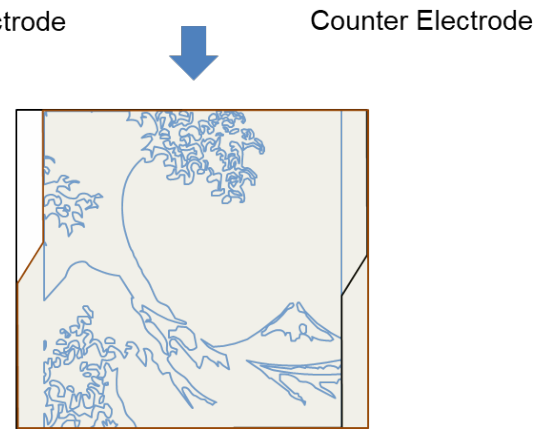

Fig. 6. The fabrication scheme of the designed ECD. An ITO film with an image depicted by scratching the ITO layer, a counter ITO film, and the stacked state.
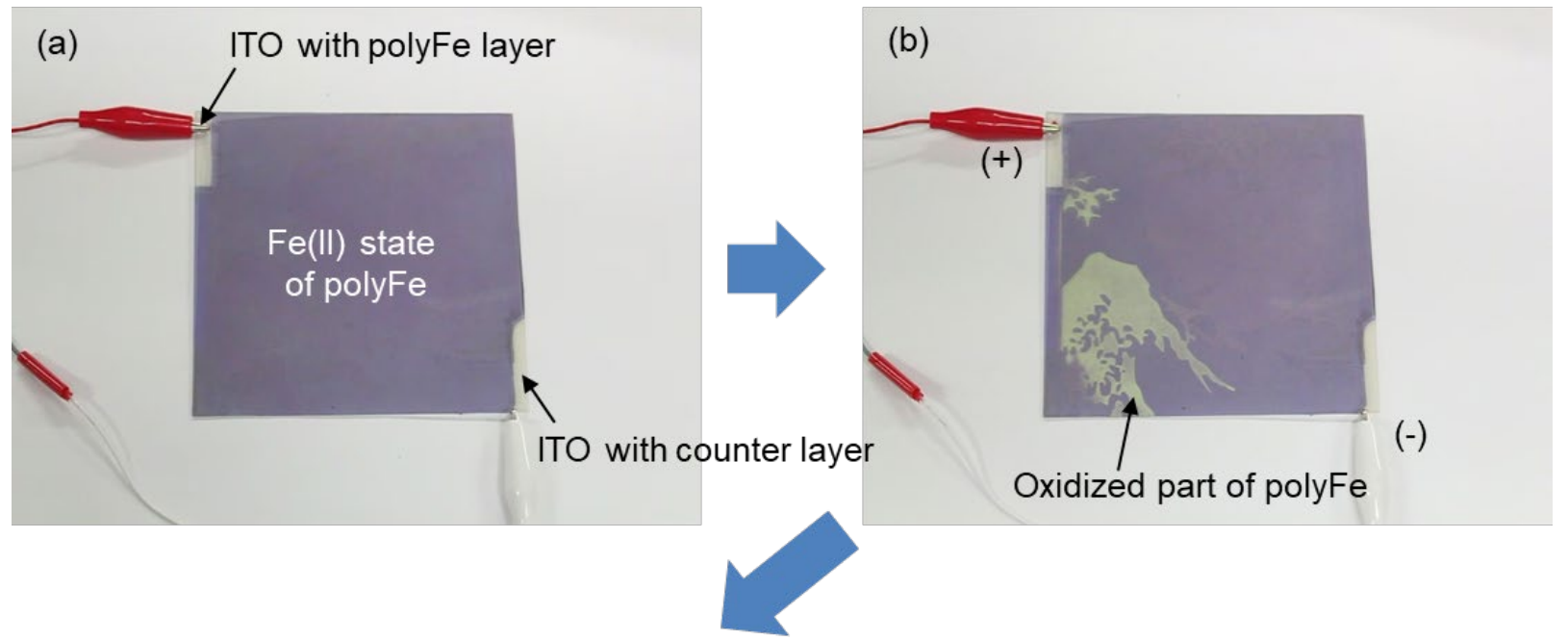

(c)

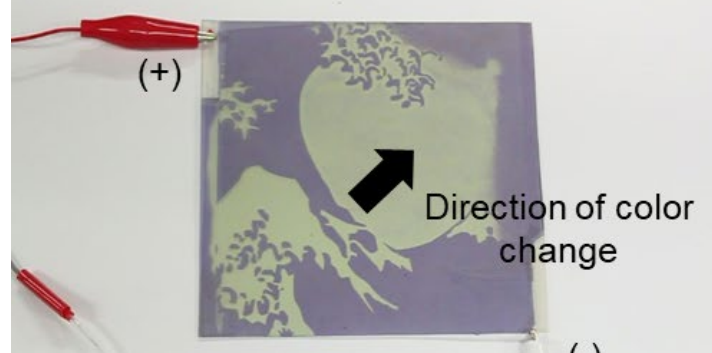

$(-)$

\subsection{Design concept}

To display an image on a flexible ECD with polyFe, an ITO film was etched mechanically using a cutting machine (Fig. 6). The ITO layer was separated to the following three area by the etched design.

(a) Totally isolated area, surrounded with the etched line

(b) Area surrounded with the etched line imperfectly, with narrow connecting path to the other area

(c) Area which is not prevented by the etched line until the left edge of the ITO layer, where the voltage is connected.

\subsection{EC behavior on the device}

It is known that polyFe slightly changes the color by exchanging the counter anion species. The color of the polyFe layer was changed from blue to blueish purple during the device fabrication because the counter anion was exchanged from acetate anion to perchlorate anion in the device (Fig. 7a). Before applying a voltage, the ECD showed the blueish purple color based on the MLCT absorption of the

(b)

(d)

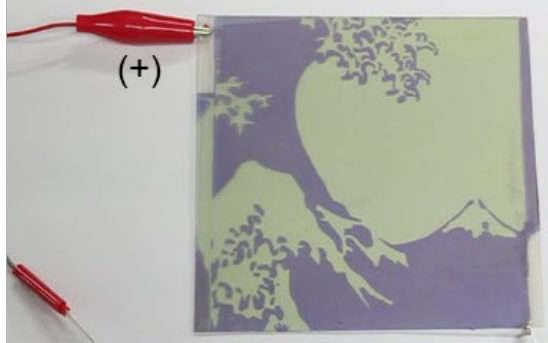

$(-)$

Fig. 7. (a) The flexible ECD with polyFe before applying a voltage. Images of the device at (b) $7 \mathrm{sec}$, (c) 30 sec, and (d) $90 \mathrm{sec}$ when $1.5 \mathrm{~V}$ was applied. 
$\mathrm{Fe}(\mathrm{II})$ complex moieties in polyFe. When the Fe(II) is oxidized to Fe(III) electrochemically, the MLCT absorption disappears and the color is changed to colorless.

When applying $1.5 \mathrm{~V}$ in the device by connecting the electrode coated with polyFe to the positive electrode side, interestingly, the color change from purple to colorless (pale green) occurred only on the left side image in the device during the first seven seconds (Fig. 7b). Then, the color change expanded from the center toward the right side, but the speed of the color change became slow. Eventually, it took $90 \mathrm{sec}$ to display the whole image after applying the voltage.

The EC behavior provided the following findings in the display system of polyFe using the mechanically etched ITO film (Fig. 8).

(i) Unlike the previously reported two display systems (Figs. 5a and 5b), the color change in the EC layer first occurs only on the near side where the battery is connected, when a narrow path of ITO is formed by the mechanical etching.

(ii) The color changes at the far side to the battery from the narrow ITO path become slow. The narrow path is considered to have high resistance. Therefore, the voltage applied between the etched electrode and the counter electrode will decrease at the far side from the narrow path. The decrease of the applied voltage between the electrode causes the slow color changes.

(iii) The color of the polyFe layer on the area of ITO surrounded with the etched line perfectly does not show the EC changes, because the electron transfer on the ITO layer is cut off by the etching.

\section{Conclusion}

A designed, flexible electrochromic (EC) display device with polyFe was successfully fabricated using a mechanically etched ITO film. The mechanical etching of an ITO film was quickly performed with a cutting machine under ambient conditions. The fabricated device showed unique changes of the display upon applying a voltage of $1.5 \mathrm{~V}$ between the two ITO electrode. This display system using the etching technique is widely available in the EC displays with the other MSPs, which show various colors or multi-color EC changes.

\section{Acknowledgement}

This work was financially supported by JST CREST (grant No. JPMJCR1533).

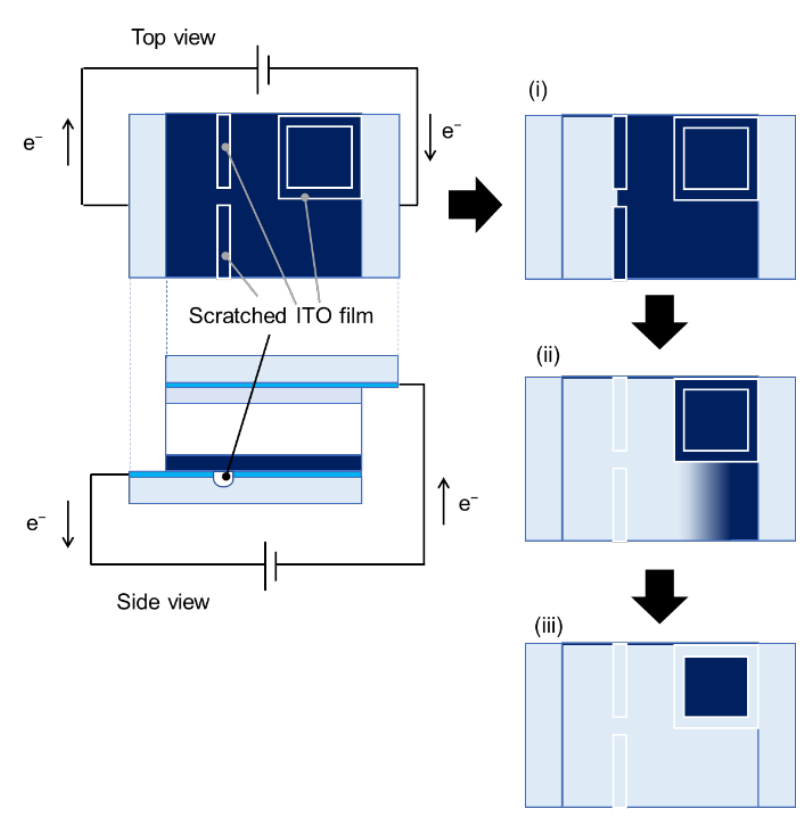

Fig. 8. Schematic images of the color changes in the ECD with polyFe using the mechanically etched ITO film. In case a narrow path of ITO and a closed area are included, the color changes occur by the order of (i) the initial change, (ii) the intermediate state, and (iii) the final state upon applying a voltage.

\section{References}

1. J. W. Xu, M. H. Chua, and K. W. Shah (eds), "Electrochromic Smart Materials", Smart Materials Series, Royal Soc. Chem. (2019).

2. C. G. Granqvist, Sol. Energy Mater. Sol. Cells, $60(2000) 201$.

3. G. A. Niklasson and C. G. Granqvist, J. Mater. Chem., 17 (2007) 127.

4. L. Groenendaal, F. Jonas, D. Freitag, H. Pielartzik, and J. R. Reynolds, Adv. Mater., 12 (2000) 481.

5. N. Kobayashi, S. Miura, M. Nishimura, H. Urano, Sol. Energy Mater. Sol. Cells, 92 (2008) 136.

6. A. Tsuboi, K. Nakamura, and N. Kobayashi, $A d v$. Mater., 25 (2013) 3197.

7. K. Takada, R. Sakamoto, S.-T. Yi, S. Katagiri, T. Kambe, and H. Nishihara, J. Am. Chem. Soc., 137 (2015) 4681.

8. P. Andersson, R. Forchheimer, P. Tehrani, and M. Berggren, Adv. Funct. Mater., 17 (2007) 3074.

9. C. Yan, W. Kang, J. Wang, M. Cui, X. Wang, C. Y. Foo, K. J. Chee, and P. S. Lee, ACS Nano, 8 (2014) 316.

10. S. Lin, X. Bai, H. Wang, H. Wang, J. Song, K. Huang, C. Wang, N. Wang, B. Li, M. Lei, and H. Wu, Adv. Mater. 29 (2017) 1703238. 
11. H.-J. Yen, C.-J. Chen, and G.-S. Liou, $A d v$. Funct. Mater. 23 (2013) 5307.

12. C. Wang, X. Jiang, P. Cui, M. Sheng, X. Gong, L. Zhang, and S. Fu, ACS Appl. Mater. Interfaces, 13 (2021) 12313.

13. R. Li, X. Ma, J. Li, J. Cao, H. Gao, T. Li, X. Zhang, L. Wang, Q. Zhang, G. Wang, C. Hou, Y. Li, T. Palacios, Y. Lin, H. Wang, and X. Ling, Nature Commun., 12 (2021) 1587.

14. G. Balamurugan, G. K. Pande, J. H. Choi, J. S. Park, Sol. Energy Mater. Sol. Cells, 216 (2020) 110714.

15. M. Higuchi, "Metallo-Supramolecular Polymers", NIMS Monographs, Springer,
Springer Nature (2019).

16. M. Higuchi, J. Mater. Chem. C, 2 (2014) 9331.

17. M. Higuchi and D. G. Kurth, Chem. Rec., 7 (2007) 203.

18. F. Han, M. Higuchi, and D. G. Kurth, J. Am. Chem. Soc., 130 (2008) 2073.

19. M. Higuchi, Y. Akasaka, T. Ikeda, A. Hayashi, and D. G. Kurth, J. Inorg. Organomet. Polym. Mater., 19 (2009) 74.

20. S. Mondal, T. Yoshida, U. Rana, M. K. Bera, and M Higuchi, Sol. Energy Mater. Sol. Cells, 200 (2019) 110000. 